ment died from their sareomas. No inhibition of tumour growth was observed with this treatment. The high mortality of the treated animals rendered it useless to continue these experiments on the basis of the dosage applied by Oberhauser et al.

F. SeElich

K. KARRER

Austrian Cancer Research Institute, Borschkegasse $8 a$,

Vienna IX.

April 18.

${ }^{1}$ Oberhauser, F. B., Croxatto, H. R., Gaillard, M. Q., and Silva, V. M., Nature, 176, 466 (1955).

\section{Innervation of the Continuously Growing Incisor}

To my knowledge, the development of the innervation of the teeth has not yet been systematically investigated. There have been sporadic remarks; for example, Holland" writes: "As far as nerve tissue is concerned, it is believed that there is no direct connection or hookup with the parent sensory nerve ganglion until the tooth erupts and until root formation is nearly completed". Different authors, for example, Power's ${ }^{2}$, in her studies of tooth innervation, has given illustrations of the rat-pulp for myelinated fibres but only from the molar teeth and illustrations of the periodontal nerve supply from the continuously growing incisor of the same species. In several animals (dog, calf, pig) I have found that in the earlier stages of the tooth development, for example, the period before the root begins to grow, there are in the pulp only unmyelinated (presumably vegetative) fibres. Later the myelinated sensory fibres appear. The pulp of the continuously growing incisor of the rat shows a similar situation. There are only unmyelinated fibres differing in size and branching. These are mainly in the neighbourhood of the vessels (see Fig. 1) and do not reach the odontoblastic layer.

Assuming that this tooth-the most important in the rat-must have an abundant nerve supply, I studied the continuously growing incisor by several methods but with the same result. Here are occasionally fibres in the incisor which resemble in their size myelinated nerve fibres, as shown in Fig. 2 ; only by close observation they reveal themselves as consisting of two or more, sometimes twisted, fibres. Conceding the possibility of there being nevertheless myelinated fibres in the incisor pulp, they must be very rare. With the same technique as used by Romanes $^{3}$, many myelinated fibres (Fig. 2) regularly showed in the molar-pulps.

In my opinion, this phenomenon can be explained as follows. The continuously growing incisor of the rat (and perhaps other rodents) behaves as an ordinary developing tooth before root formation, so far as innervation is concerned. The fact that the labial side of this tooth is covered with enamel up to the basal end means virtually that this tooth consists only of a crowntube. Both the developing ordinary tooth of the higher mammals and the continuously growing incisor are lacking in a sensory nerve supply. The difference is only that in the former case this is transitory and in the latter it is the permanent physiological status. Common for both is the fact that the tooth itself is in a developing state. The physiological foundation of this type of innervation is, in my opinion, as follows:

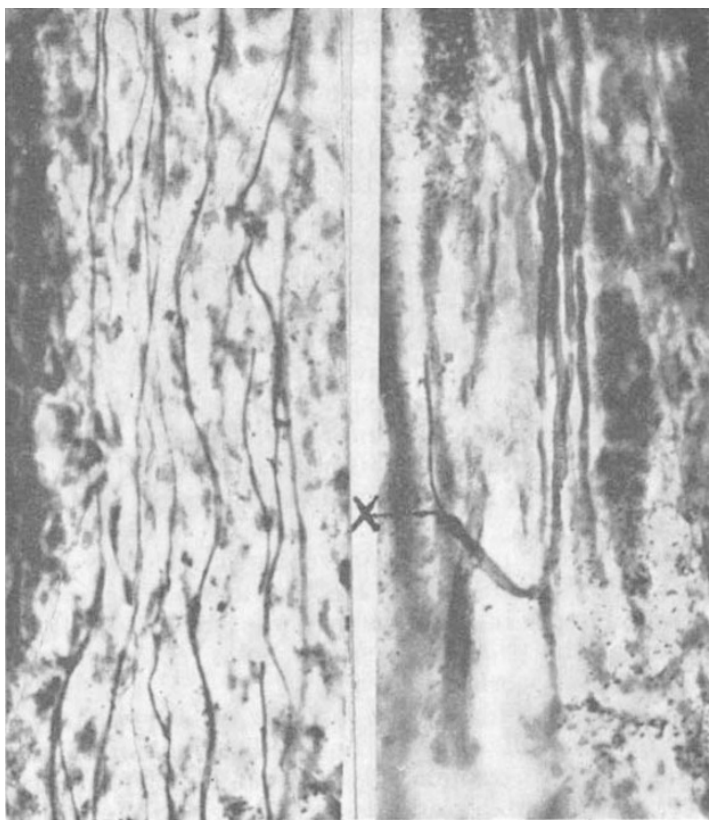

(1)

(2) Fig. 1. From the pulp of the continuously growing incisor of the
rat. Unmyelinated nerve fflbres. Silver staining (ref. 3). rat. Unmyelinated nerve $(\times 500)$

Fig. 2. From the puip-horn of the rat molar (same animal as in Fig. 1). Myelinated nerve flbres; at $X$ the nerve flbre loses its myelin sheet. Silver staining (ref. 3). ( $\times 500)$

the continuously growing incisor is wearing out by the physiological abrasion. The pulp-cavity or pulp-tube is by this abrasion slowly closed by irregular dentine production which is different from the so-called. secondary dentine of the molars and resembles a degenerative calcification. The former often has an irregular structure with many holes in it. Despite this, a pathological process, for example, inflammation, very seldom occurs in the incisor of the rat. This fact may be connected with the above-mentioned absence or scarcity of the sensory nerve supply.

D. Hattyasy

Clinic for Dental and Oral Diseases,

University Medical School, Szeged.

${ }^{1}$ Holland, D. J., Amer. J. Orthou., 41, 27 (1955).

2 Powers, Marg. M., J. Dent. Res., 31, 383 (1952).

${ }^{3}$ Romanes, G. J., J. Anat., 87, 107 (1950), and personal communication

\section{Inexcitability of Cortical Dendrites to Electric Stimuli}

A PRominent component of the electrical activity of the cerebral cortex is the surface negative potential lasting about $15 \mathrm{~m}$.sec. It is evoked synaptically by stimulation of various corticipetal pathways (for example, the callosal fibres or optic radiations), antidromically on stimulating the corticifugal pyramidal axons, or by stimuli directly applied to the cortex. This long-lasting surface negative potential has been ascribed, on good experimental grounds, to electrogenic activity in the apical dendrites of pyramidal cells ${ }^{1}$.

Intravenous injection of $d$-tubocurarine $(0 \cdot 5-$ $3 \mathrm{mgm}$./kgm. cat weight) into the succinylcholineparalysed preparation diminishes and temporarily 